\title{
Nueva generación de Stents, con polímero bio-compatible: resultados y seguimiento clínico.
}

\author{
Carlos Caorsi S. ${ }^{1}$, Victoria Barra R.1, ${ }^{\text {, }}$ Ronald Kauffmann Q.1 ${ }^{1}$ Fernando Pineda A. ${ }^{1}$ \\ ${ }^{1}$ Laboratorio Cardiovascular Invasivo. Clínica Las Condes, Santiago. \\ ${ }^{2}$ Tecnólogo Médico
}

\section{Resumen}

Introducción: Una nueva generación de Stents medicados (DES) polímeros bio-compatibles no trombogénicos permitirían una rápida re-epitelización, reduciendo la inflamación y manteniendo la función endotelial de la pared vascular. Por lo tanto, no solo la droga anti-proliferativa del DES, determina la respuesta de la pared vascular sino que la biocompatibilidad del polímero también juega un rol fundamental. Objetivo: Evaluar el resultado intrahospitalario y seguimiento clínico de los pacientes sometidos a angioplastía coronaria electiva con un DES con polímero bio-compatible.

Método: Se estudiaron 52 pacientes sometidos a angioplastía con DES, durante un período de seguimiento entre 3 y 15 meses (x 8.6 meses). Se evaluó, MACE definido como muerte, infarto miocárdico, cirugía urgencia, revascularización repetida de lesión objetivo (TLR), a los 30 días, 3, 6, 9 y 12 meses. Además se evaluó la trombosis del Stent, 0-30 días (precoz), 31-360 días (tardía), más de 361 días (muy tardía).

Resultados: La edad promedio del grupo fue de $63.8 \pm 10.8$ años, hipertensión arterial $71.15 \%$, diabetes 17.3\%, dislipidemia $50 \%$, tabaquismo 28.8\%. Un 65.3\% de los pacientes tenían enfermedad de 2 o 3 vasos, con un $51.9 \%$ de compromiso de la arteria descendente anterior (ADA). El 61.2\% de las lesiones eran lesiones B-2 o C (clasificación American Heart Association). Pre procedimiento el diámetro de referencia del vaso (RVD), fue de $3.0 \pm 0.6 \mathrm{~mm}$, con un diámetro luminal mínimo (MLD) de $0.8 \pm 0.5 \mathrm{~mm}$, un porcentaje de estenosis de $77.2 \pm 17.9 \%$ y el largo promedio de las lesiones fue de $14 \pm 8.3 \mathrm{~mm}$. El éxito del dispositivo y del procedimiento fue de $98.1 \%$. Durante el período de seguimiento el MACE fue $3.8 \%$ y no se registraron trombosis de stents.

Conclusiones: Durante el período de estudio, se observó una tasa baja de MACE relacionado al procedimiento, sin trombosis probable, posible, o definitiva. El resultado clínico y seguimiento confirman el buen desempeño y seguridad de este stent con polímero bio-compatible.

Palabras Claves: Angioplastía con stent, stent medicados, enfermedad coronaria.

\section{New generation of biocompatible polymer coated stents: immediate and follow-up results in 52 patients.}

Background: a new generation of drug-eluting stents (DES) coated with a non-thrombogenic and biocompatible polymer allows a fast re-epithelization, reducing inflammation and maintaining endothelial function of the vascular wall. Therefore, not only the anti-proliferative effect of the drug contained in DES

Correspondencia: Dr. Carlos Caorsi S.

Clínica Las Condes

Lo Fontecilla 441 - Las Condes

Correo Eléctronico: ccaorsi@clinicalascondes.cl 
determines the response of the vascular wall, but also the polymer biocompatibility seems to play a fundamental role.

Aim: to evaluate the results and clinical follow up of patients submitted to elective coronary angioplasty using a biocompatible polymer coated DES.

Method: 52 patients treated with DES were followed from 3 to 15 months (mean 8.6 months) after stent implantation. Overall MACE defined as, death, myocardial infarction, emergent cardiac surgery or repeat revascularization of the target lesion (TLR) was evaluated at 30 days, 3, 6, 9 and 12 months. Stent thrombosis was also evaluated at 0-30 days (early), 31-360 days (late), and more than 361 days (very late).

Results: Mean age was $63.8 \pm 10.8$ years; hypertension was present in $71.1 \%$, diabetes in $17,3 \%$, hyperlipidemia in $50 \%$, and $28.8 \%$ were current smokers. Two or three vessel diseases were present in $65.3 \%$ of patients: lesions were present in the LAD in 51.9\%. Lesions were classified as B-2 or C (according to American Heart Association) in $61.2 \%$ of cases. Pre procedure reference vessel diameter (RVD) was $3.0 \pm 0.6 \mathrm{~mm}$, with a minimum luminal diameter (MLD) of $0.8 \pm 0.5 \mathrm{~mm}$, percentage stenosis of $77.2 \pm 17.9 \%$ and lesion length averaged $14 \pm 8.3 \mathrm{~mm}$. Both device and procedure success rates were $98.1 \%$. During follow up MACE occurred in $3.8 \%$ and no stent thrombosis were registered.

Conclusion: During the study period, the observed procedure related MACE rate was low, with no instances of probable, possible, or definitive stent thrombosis. The clinical results confirmed the good performance and security of this biocompatible polymer containing stent.

Keywords: Drug eluting stents, biocompatible polymer stents

Recibido el 22 de junio de 2009, aceptado el 28 de octubre de 2009

Rev Chil Cardiol 2009; 28: 357-362

\section{Introducción}

El uso de stents medicados (DES) en lugar de stents metálicos (BMS), en las intervenciones coronarias percutáneas ha determinado una significativa reducción de la tasa de re-estenosis angiográfica1-3. Los DES están compuestos por el stent metálico, la droga y un sistema de entrega o liberación de ésta, constituido por un polímero. La primera generación de DES se desarrollaron con polímeros bio-estables que permanecían en el organismo hasta después que la droga se disipaba. Sin embargo, si este polímero no es bio-compatible, puede generar una importante respuesta inflamatoria a nivel de la pared vascular alterando la re-endotelización y determinando mayor riesgo de trombosis. Por lo tanto, un polímero ideal no debiera desencadenar una gran respuesta inflamatoria, como la unión de monocitos y la liberación de factores quimiotácticos y citoquinas. Un polímero ideal debe ser bio-compatible, hidrofílico, no inflamatorio, no trombogénico, y permitir una endotelización rápida, completa y funcional. Se ha propuesto en la literatura que la hipersensibilidad a los polímeros con pobre bio-compatibildad podría ser responsable de una reepitelización alterada y trombosis del stent tardía4-7. Se han realizado grandes esfuerzos por investigar la interacción entre la droga y el polímero, así como también el rol de la capa de polímero residual en la determinación del resultado clínico, tanto en términos de eventos adversos (re-estenosis \& trombosis), como el resultado a largo plazo ${ }^{8}$.

Recientemente se desarrollo un nuevo DES, con polímero bio-compatible (BioLinx) (Fig. 1), el cual permite una endotelización rápida (28 días) y funcional (Fig. 2).

Con el objetivo de evaluar el resultado clínico y seguimiento con este nuevo dispositivo se enroló a pacientes sometidos a angioplastíia con DES con polímero bio-compatible (BioLinx) en un único centro. 


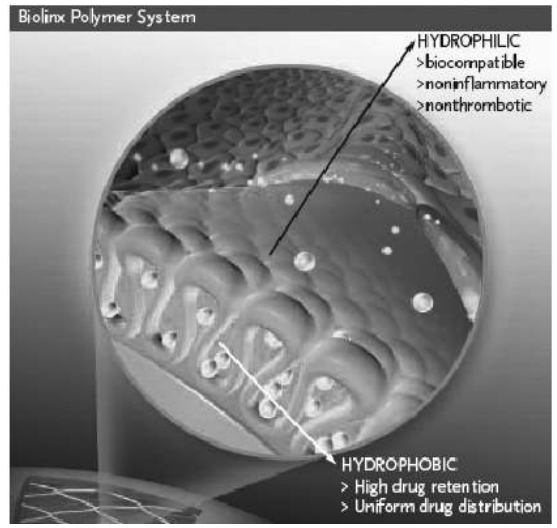

Figura 1: Bases biofísicas del polímero bio-compatible BioLinx.

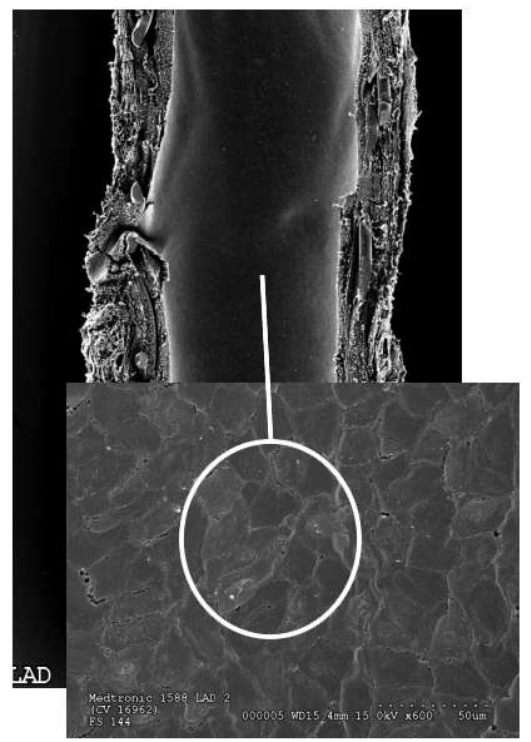

Figura 2: Arteria de porcino a los 28 días (LAD), FS144 Representative SEM

\section{Material y Método}

Este estudio se realizó sólo en un centro, es prospectivo, no-randomizado, de un sólo brazo, con uso del stent Endeavor Resolute para tratar 52 pacientes con enfermedad coronaria isquémica atribuible a estenosis significativas de las arterias coronarias nativas. El seguimiento clínico de estos pacientes se prolongó desde los 3 meses hasta los 15 meses (promedio 8.6 meses), todos los pacientes consintieron este seguimiento. Los datos se obtuvieron y el seguimiento se realizó según el registro de angioplastías del Laboratorio Cardiovascular Invasivo de Clínica las Condes. Se consideraron elegibles pacientes con enfermedad multi-vasos y de vaso único. En el $88 \%$ de los casos el acceso vascular fue femoral. Sólo lesiones con un diámetro de estenosis $\geq 50 \%$ y con largos $\geq 10 \mathrm{~mm}$, vasos con diámetros de referencia $\geq 2.25 \mathrm{a} \leq 3.5$ fueron incluidos. Los pacientes recibieron al menos aspirina $100 \mathrm{mg}$. antes del procedimiento y continuada indefinidamente post-procedimiento. Se cargaron con clopidogrel $\geq 300 \mathrm{mg} 24$ horas antes del procedimiento o inmediatamente luego de la implantación del stent. La 
terapia se mantuvo por al menos 6 meses post procedimiento. La trombosis de los stents fue evaluada de acuerdo a la definición de la Academic Research Consortium (ARC), 0-30 días (precoz), 31-360 días (tardía), más de 361 días (muy tardía). Los "endpoints" primarios incluyeron eventos cardíacos mayores (definidos como muerte, infarto al miocardio, cirugía cardíaca de urgencia, revascularización de la lesión objetivo (TLR clínico), es decir MACE, a los 30 días, 3, 6, 9 y 12 meses. También se evaluó el éxito agudo del dispositivo y del procedimiento.

Las mediciones angiográficas fueron realizadas con un cineangiógrafo Siemens (Syngo anál. vasc. coronario angiografía cuantitativa (QCA)) antes y pos-procedimiento. Se obtuvo consentimiento informado de todos los pacientes.

\section{Resultados}

Las características demográficas basales de los pacientes se detallan en la tabla 1.

\section{Datos Demográficos}

\begin{tabular}{ll}
\hline Masculinos (\%) & 82,3 \\
Edad (años) & $63,8 \pm 10,8$ \\
HTA (\%) & 71,15 \\
Diabetes (\%) & 17,3 \\
Dislipidemia (\%) & 50 \\
Tabaquismo (\%) & 28,8 \\
Cirugía previa (\%) & 11,5 \\
PTCA previa (\%) & 25 \\
\hline
\end{tabular}

Tabla 1: HTA. hipertensión arterial; PTCA, angioplastía

El sexo, la distribución y los perfiles de factores de riesgo de la población estudiada son consistentes con una población de pacientes que presentan síntomas de isquemia debido a lesiones significativas en arterias nativas.

Según indica la Tabla 2, un 65,3\% de los pacientes tenían enfermedad de 2 o 3 vasos, un $51.9 \%$ de los casos presentaban compromiso de la arteria descendente anterior (ADA) (Fig. 3a). El 61.2\% de las lesiones eran lesiones B-2 o C, mediana a alta complejidad (clasificación American Heart Association). Seis pacientes habían sido sometidos a revascularización miocárdica (by-pass) y 13 a angioplastía previa.

Pre procedimiento el diámetro de referencia del vaso (RVD), fue de $3.0 \pm 0.6 \mathrm{~mm}$, con un diámetro luminal mínimo (MLD) de $0.8 \pm 0.5 \mathrm{~mm}$, un porcentaje de estenosis de $7.2 \pm 17.9 \%$ y el largo promedio de las lesiones fue de $14 \pm 8.3 \mathrm{~mm}$.

En un $95 \%$ de las angioplastías se predilató con balón y en sólo 5 stents se utilizó la técnica de stenting directo. En 52 pacientes se utilizaron 98 stents, 1.88 stent/paciente. El éxito agudo del dispositivo y del procedimiento fue de $98.1 \%$. El MACE a 30 días fue $3.8 \%$, con un paciente que elevó marca-dores enzimáticos 5 veces el valor normal post procedimiento y se calificó como infarto agudo al miocardio (IAM) no $Q$. Un paciente fue enviado a cirugía por angina persistente en relación a un vaso no relacionado, con oclusión total crónica que no se logró revascularizar.

Un paciente debió suspender el Plavix y la aspirina antes de un mes producto de una hemorragia digestiva secundaria a un cáncer gástrico, no ha presentado eventos adversos cardiovasculares. Durante el período de seguimiento no se han presentado eventos cardiovasculares mayores por lo que el MACE se ha mantenido y no se registraron trombosis de stents. 
Nueva generación de Stents, con polímero bio-compatible: resultados y seguimiento clínico.

\section{Características de la lesión y el procedimiento}

\begin{tabular}{lc} 
& \\
\hline ADA (\%) & 51,9 \\
Enf. 2-3 vasos (\%) & 65.3 \\
Lesión B2 - C (\%) & 61.2 \\
RVD (mm) & $3.0 \pm 0.6$ \\
MLD (mm) & $0.8 \pm 0.5$ \\
Estenosis (\%) & $77.2 \pm 17.9$ \\
Largo Lesión (mm) $^{\text {Exito dispositivo (\%) }}$ & $14.0 \pm 8.3$ \\
Exito procedimiento (\%)\# $^{*}$ & 98.1 \\
Exito lesión (\%) $^{\dagger}$ & 98.1 \\
\end{tabular}

Tabla 2: ADA, arteria descendente anterior; RVD, Diámetro del vaso de referencia; MLD, Diámetro luminal mínimo.

* Éxito dispositivo definido como $<50 \%$ estenosis residual final en el segmento; \# Éxito procedimiento definido como $<50 \%$ estenosis residual final sin eventos cardiacos mayores en 30 días; † Éxito lesión definido como $<50 \%$ estenosis residual final en el segmento.
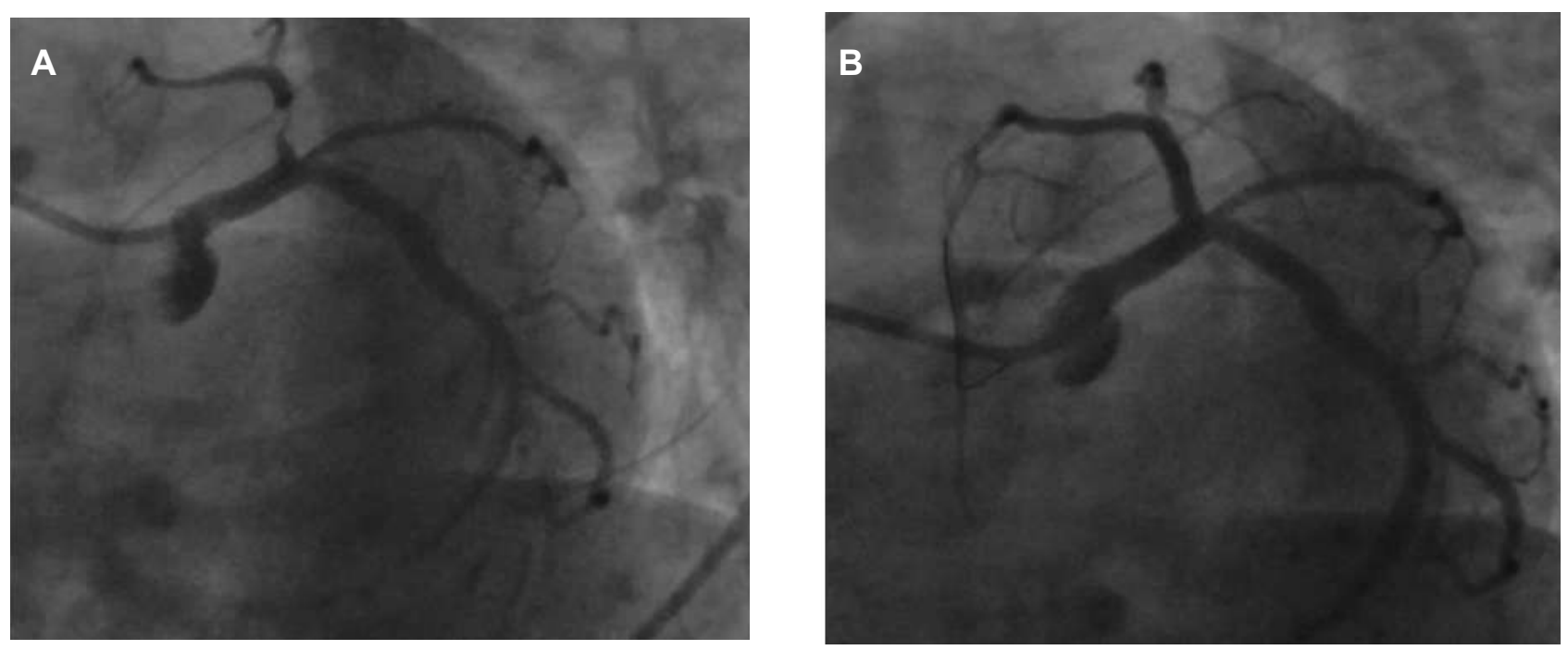

Figura 3: Caso de lesión ADA proximal. a) Lesión crítica. b) Pos implantación de stent.

\section{Discusión}

Si bien el grupo de pacientes es pequeño, se puede concluir que la mayoría son pacientes con enfermedad de 2 y 3 vasos, con compromiso significativo de la ADA Fig. 3 y con lesiones moderadamente complejas. En este grupo representativo de pacientes con enfermedad coronaria moderada, se observó una tasa baja de MACE relacionado al procedimiento, sin trombosis probable, posible, o definitiva, durante el período de estudio. También se observó una baja necesidad de revascularización quirúrgica en esta población de pacientes moderadamente complejos.

El diseño de un stent debe considerar tres elementos básicos, la plataforma, el polímero y la droga. Son estos elementos los que determinan finalmente la biocompatibilidad y respuesta fisiológica de la pared vascular. Después de la implantación de un stent la reparación vascular y re-endotelización es un 
proceso complejo y de duración variable. La infusión de acetilcolina a los 28 días de implantado este stent bio-compatible en arterias de porcino presentan una expresión normal del ácido ribonucleico, mensajero de la óxido nítrico sintetasa (mRNA de eNOS), demostrando que las propiedades endotelio-vasculares, se mantienen intactas. (Datos en archivos de Medtronic Inc.) Uno de los objetivos es conseguir un equilibrio entre el TLR y la posible trombosis del stent. En este caso se logra que el $85 \%$ de la droga se libere a los 60 días y se alcanza la completa reepitelización a los 120 días (Fig. 2).

El Endeavor Resolute Clinical Trial con 130 pa- cientes demostró no TLR, no revascularización del vaso objetivo (TVR) y una tasa de MACE de sólo $7 \%$. Un índice de pérdida tardía (late loss index) intra stent de $0.12 \pm 016$ y de $0.08 \pm 021$ intrasegmento a 12 meses $^{9}$.

Estos resultados al igual que el comportamiento clínico y seguimiento de nuestros pacientes confirman el buen desempeño de este stent con polímero bio-compatible, indicando que es un dispositivo seguro con una baja tasa de eventos clínicos. En los próximos meses tendremos los resultados de los estudios resolute que en global enrolaran un significativo número de pacientes.

\section{Referencias}

1. KATRITSIS DG, KARVOUNI E, IOANNIDIS JP. Meta-analysis comparing drug-eluting stents with bare metal stents. Am J Cardiol. 2005; 95: 640-643.

2. STONE GW, MOSES JW, ELLIS SG, SCHOFER J, DAWKINS KD, MORICE MC, et al. Safety and efficacy of sirolimusand placlitaxel-eluting coronary stents. N Engl J Med. 2007; 356: 998-1008.

3. KASTRATI A, MEHILLI J, PACHE J, KAISER C, VALGIMIGLI $\mathrm{M}, \mathrm{KELBAEK} \mathrm{H}$, et al. Analysis of 14 Trials comparing sirolimus-eluting stents with bare-metal stents. N Engl J Med. 2007; 356: 1030-1039.

4. NEBEKER JR, VIRMANI R, BENNETT CL, HOFFMAN JM, SAMORE MH, ALVAREZ J, et al. Hypersensitivity cases associated with drug -eluting coronary stents: a review of available cases from the research on adverse drug events and reports (RADAR) project. J Am Coll Cardiol. 2006; 47: 175-81.

5. JONER M, FINN AV, FARB A, MONT EK, KOLODGIE FD, LADICH E, et al. Pathology of drug-eluting stents in humans: delayed healing and late thrombotic risk. J Am Coll Cardiol. 2006; 48: 193-202.
6. LUSCHER TF, STEFFEL J, EBERLI FR, JONER M, NAKAZAWA G, TANNER FC, et al. Drug-eluting stent and coronary thrombosis: biological mechanisms and clinical implications. Circulation 2007; 115: 1051-8.

7. FINN AV, KOLODGIE FD, HARNEK J, GUERRERO LJ, ACAMPADO E, TEFERA $K$, et al. Differential response of delayed healing and persistent inflammation at sites of overlapping sirolimus- or paclitaxel-eluting stents. Circulation. 2005 12; 112: 270-8.

8. CARTER A, AGGARWAL M. Delivery Systems for Stents: Are There Clinical Implications of the 'Polymer' Debate? Emerging Technology. American Journal of Drug Delivery. 2003; 1: 157.

9. MEREDITH I. RESOLUTE clinical trial, a first-in-man study evaluating the new Endeavor RESOLUTE zotarolimus-eluting stent system. Cardiovascular Research Foundation's eighteenth annual Transcatheter Cardiovascular Therapeutics (TCT) scientific symposium in Washington, D.C. 2006. 